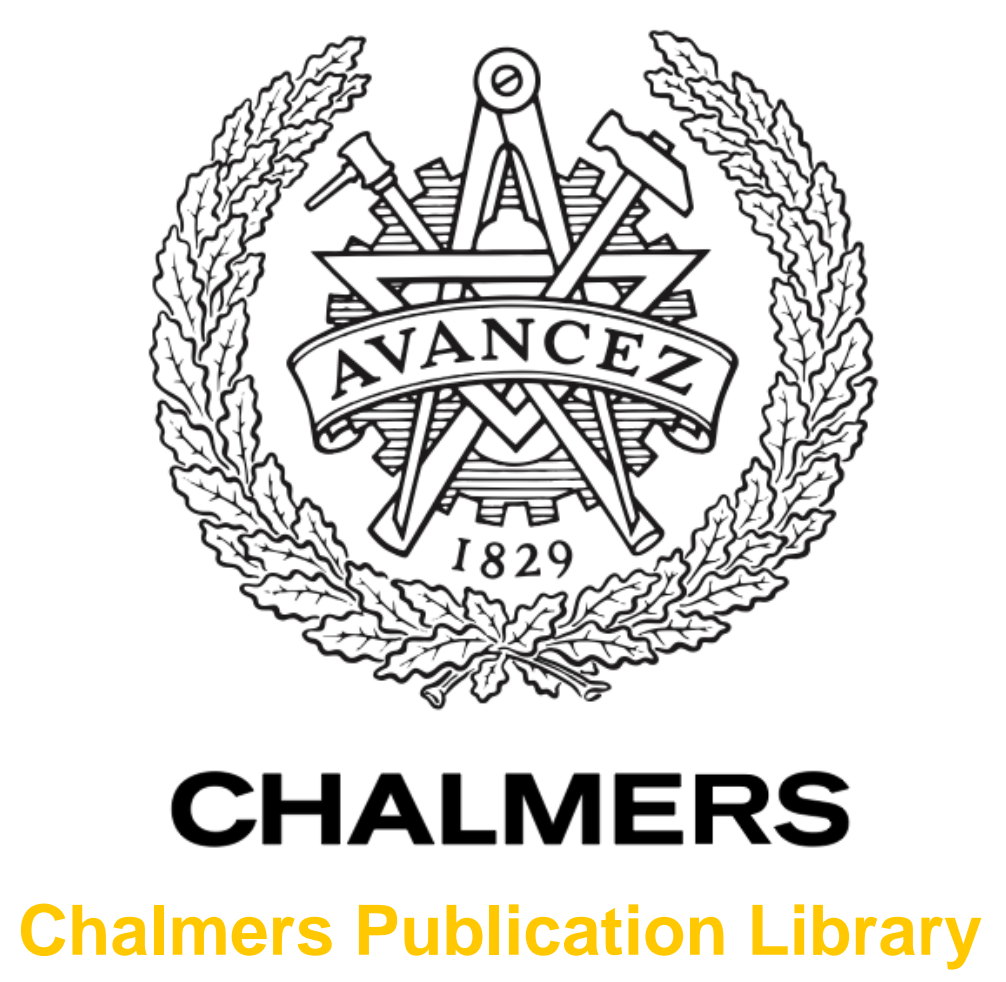

Effects of noise on tomographic breast imaging

This document has been downloaded from Chalmers Publication Library (CPL). It is the author's version of a work that was accepted for publication in:

30th URSI General Assembly and Scientific Symposium, URSIGASS 2011, Istanbul, 13-20 August 2011

Citation for the published paper:

Zeng, X. ; Fhager, A. ; Persson, M. (2011) "Effects of noise on tomographic breast imaging". 30th URSI General Assembly and Scientific Symposium, URSIGASS 2011, Istanbul, 13-20

August 2011

http://dx.doi.org/10.1109/URSIGASS.2011.605137

2 .

Down「oaded from: http://publications.lib.chalmers.se/publication/148119

Notice: Changes introduced as a result of publishing processes such as copy-editing and formatting may not be reflected in this document. For a definitive version of this work, please refer to the published source. Please note that access to the published version might require a subscription.

Chalmers Publication Library (CPL) offers the possibility of retrieving research publications produced at Chalmers University of Technology. It covers all types of publications: articles, dissertations, licentiate theses, masters theses, conference papers, reports etc. Since 2006 it is the official tool for Chalmers official publication statistics. To ensure that Chalmers research results are disseminated as widely as possible, an Open Access Policy has been adopted.

The CPL service is administrated and maintained by Chalmers Library. 


\title{
Effects of Noise on Tomographic Breast Imaging
}

\author{
Xuezhi Zeng ${ }^{1}$, Andreas Fhager ${ }^{2}$, and Mikael Persson ${ }^{3}$ \\ ${ }^{1}$ Department of Signals and Systems, Chalmers University of Technology, 41296 Gothenburg, Sweden. \\ xuezhi@chalmers.se \\ ${ }^{2}$ Department of Signals and Systems, Chalmers University of Technology, andreas.fhager@chalmers.se \\ ${ }^{3}$ Department of Signals and Systems, Chalmers University of Technology, mikael.persson@chalmers.se
}

\begin{abstract}
We study the effect of noise on microwave breast imaging by numerical simulations. A high contrast breast model is considered and the image reconstructions are carried out in time domain using a nonlinear inversion algorithm. A relative reconstruction error is defined in order to quantitatively evaluate the image distortion due to noise. The relative reconstruction errors of noisy reconstructions are obtained when different levels of amplitude and phase errors are taken into account.
\end{abstract}

\section{Introduction}

Breast tumor detection using microwaves has attracted considerable interests in the last few years [12]. Microwave breast imaging relies on the contrast in dielectric properties between the normal tissue and malignant tumors. Previous studies showed that the dielectric contrast between cancerous and normal breast tissues was as high as more than three times [3-4]. Recently, it has been suggested that the contrast in dielectric properties between the malignant tumor and normal breast tissue varies considerably depending on the compositions of the breast [5].

There are two main approaches for microwave breast imaging: microwave tomography [6-7] and radar based imaging [8]. In both approaches, microwave signals are used to illuminate the breast and scattered signals are received at numerous locations for the use in image reconstructions. With microwave tomography, the dielectric property distributions of the breast are reconstructed quantitatively from the received data by using nonlinear inversion algorithms. Radar based methods avoid complicated reconstruction algorithm, in the aim of providing qualitative pictures of strong scatters (e.g. tumor) in the breast.

Microwave measurements for breast imaging are subject to noise, which may influence the detection of breast tumor. As little concern has been paid to this aspect, in this paper, we study the influence of noise on breast tumor detection using the microwave tomography technique. The deterioration of reconstruction quality of a high contrast breast model due to noise are evaluated quantitatively for different levels of amplitude and phase errors.

\section{Microwave Tomography}

Figure 1 shows the measurement configuration of a typical microwave tomography system. An objectunder-test (OUT) is surrounded by an antenna array. Microwave signals are transmitted sequentially by the antennas into the OUT, and the reflected and transmitted signals are received by the remaining antennas. This process will be repeated until all the antennas have been transmitted. Therefore, if $M$ is the number of the antennas, totally $M \times(M-1)$ data sets are recorded. The received data is then utilized for reconstructing the spatial distribution of the dielectric properties of the OUT.

In our approach, the transmitted signals are ultra-wideband (UWB) signals and the image reconstructions are performed in time domain. The dielectric properties of the object are recovered by comparing the measured signals with the computed signals obtained using finite-difference time-domain (FDTD) method. 


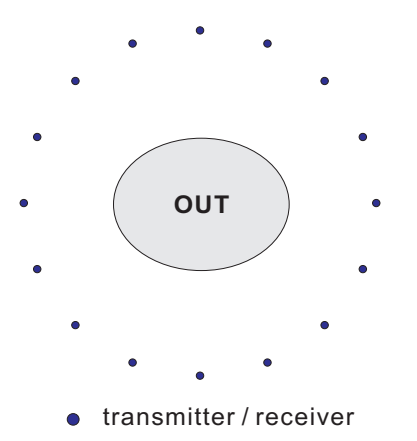

Figure 1: Measurement configuration for microwave tomography.

A cost function $F$ containing the difference between the measured data and computed data is defined below:

$$
F(\epsilon, \sigma)=\int_{0}^{T} \sum_{m=1}^{M} \sum_{n=1}^{M-1}\left(\left|\mathbf{E}_{m}^{\text {simu }}\left(\epsilon, \sigma, \mathbf{R}_{n}, t\right)-\mathbf{E}_{m}^{\text {meas }}\left(\mathbf{R}_{n}, t\right)\right|^{2}\right) d t,
$$

Here $\epsilon$ and $\sigma$ are the permittivity and conductivity values of the object, $\mathbf{E}_{m}^{\operatorname{simu}}\left(\epsilon, \sigma, \mathbf{R}_{n}, t\right)$ is the calculated field in FDTD and $\mathbf{E}_{m}^{\text {meas }}\left(\mathbf{R}_{n}, t\right)$ is the measured data. $M$ is the number of antennas. In the reconstruction procedure, a conjugate-gradient optimization is used to iteratively update the dielectric properties of the object in order to minimize the cost function $F$. A detailed description of the time domain inversion algorithm can be found in [7].

\section{Numerical Studies}

As nonlinear reconstruction methods are proposed specifically for imaging high contrast objects, a high contrast breast model is considered here.

Figure 2(a) shows the breast model and the antenna array configuration used in the simulations. The model consists of a $2 \mathrm{~mm}$ skin layer, a circular healthy tissue region with radius of $48 \mathrm{~mm}$ and two tumors with radius of $5 \mathrm{~mm}$. The dielectric properties are assumed to be frequency independent and the permittivities and conductivities for skin, healthy tissue, and tumor are: $\epsilon_{\text {skin }}=40, \sigma_{\text {skin }}=1 \mathrm{~S} / \mathrm{m} \mathrm{[4]} ; \epsilon_{\text {breast }}=10$, $\sigma_{\text {breast }}=0.2 \mathrm{~S} / \mathrm{m}[5] ; \epsilon_{\text {tumor }}=55, \sigma_{\text {tumor }}=1 \mathrm{~S} / \mathrm{m}[5]$. Sixteen antenna elements are equally spaced at the perimeter of a $19 \mathrm{~cm}$ diameter circle and immersed in a matching liquid with permittivity $\epsilon_{\text {liquid }}=12$ and conductivity $\sigma_{\text {liquid }}=0.2 \mathrm{~S} / \mathrm{m}$.

The measured scattering data was numerically generated by using FDTD. Each time one antenna was selected for transmitting the signal into the breast and the scattered signal of the breast was then recorded by the remaining antennas. This process was repeated and totally $16^{*} 15$ groups of data were obtained. The obtained data were then utilized to reconstruct the dielectric properties by using the time domain inversion algorithm. The conductivity reconstruction has worse quality than the permittivity reconstruction and only the latter is presented here.

\subsection{Noise Free Reconstruction}

A multiple-step-reconstruction strategy was used for enhancing the reconstruction quality [7]. Figure 2 (b) shows the permittivity profile of the breast reconstructed from noise free data. The grid cell size was set to be $1 \mathrm{~mm}$ in the forward simulation and $2 \mathrm{~mm}$ in the inverse reconstruction. The reconstructed region was $120 \mathrm{~mm} \times 120 \mathrm{~mm}$. The color bar on the right side of the image indicates the permittivity values. 


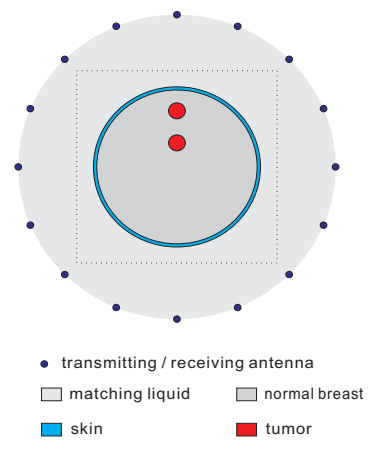

(a)

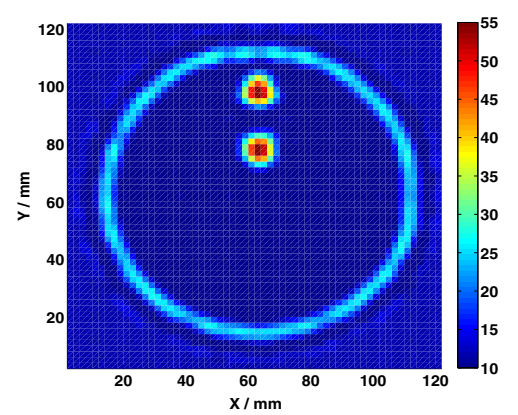

(b)

Figure 2: (a) the breast model considered in the simulation and (b) the noise free reconstruction.

It can be seen that the objects are on the correct positions, with dimensions corresponding well to the original model. A high spatial resolution is achieved and the permittivities of the tumors are well consistent with the values presented in the model. The skin profile is resolved very well, although the reconstructed permittivity is lower than the real value.

\subsection{Noisy Reconstructions}

Using the same reconstruction settings as for the noise free reconstruction in Figure 2 (b), we took different levels of amplitude errors and phase errors into account in the reconstruction process. Both the amplitude and phase errors were modeled as zero-mean normal distribution random process. In order to quantitatively evaluate the influence of the noise on the reconstruction quality, a relative reconstruction error was defined below:

$$
\delta_{\text {rec }}=\frac{\iint_{S}\left[\epsilon_{\text {rec }}^{\text {noisy }}(x, y)-\epsilon_{\text {rec }}^{\text {noisefree }}(x, y)\right]^{2} \cdot d s}{\iint_{S}\left[\epsilon_{\text {rec }}^{\text {noisefree }}(x, y)\right]^{2} \cdot d s},
$$

where $\epsilon_{\text {rec }}^{\text {noisy }}$ and $\epsilon_{\text {rec }}^{\text {noisefree }}$ were the permittivity profiles reconstructed from noisy and noise free data. $S$ was the 2-D reconstructed region.

Figure 3 (a) and (b) give the relative reconstruction errors of the noisy reconstructions when the amplitude error and the phase error are considered separately. The results are the average value from five repetitive simulations. It can be seen that when the signal-to-noise ratio (SNR) of the scattering data is higher than 16 $\mathrm{dB}$, the relative reconstruction error changes very slowly with the SNR. As the SNR decreases further, the changing rate of the relative reconstruction error versus the SNR becomes higher. The presented results also indicate that the influence of a phase error of 30 degrees on the reconstruction quality is very similar to that caused by an amplitude error with SNR about $11 \mathrm{~dB}$. Figure 4 (a) and (b) are two images reconstructed from noisy data with the SNR of $15 \mathrm{~dB}$ and the phase error of 10 degrees respectively. The relative reconstruction errors of these two images are respectively $0.13 \%$ and $0.12 \%$. Comparing these two images with the noise free reconstruction, it can be seen that the image distortion is very small.

\section{Conclusion}

The effect of noise on tomographic breast imaging is numerically studied. The results suggest that the image reconstruction of the breast model by using the time domain inversion algorithm can tolerate an amplitude error with SNR about $15 \mathrm{~dB}$ and a phase error of around 10 degrees. This work is of importance for the design of an accurate breast cancer detection system. The study is based on microwave tomography technique and a future work can be an investigation based on radar based imaging. 


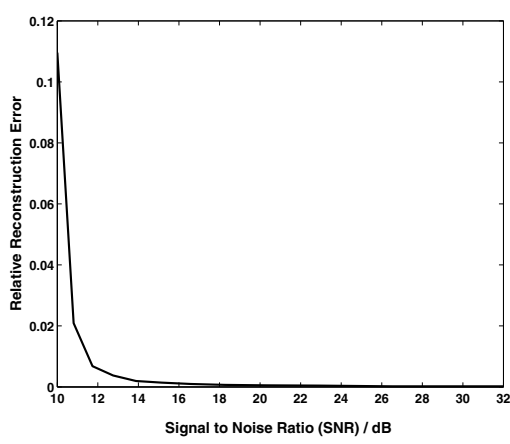

(a)

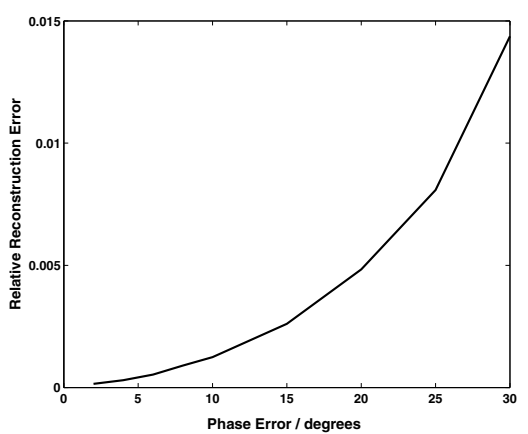

(b)

Figure 3: Relative reconstruction errors versus (a) the amplitude error and (b) the phase error.

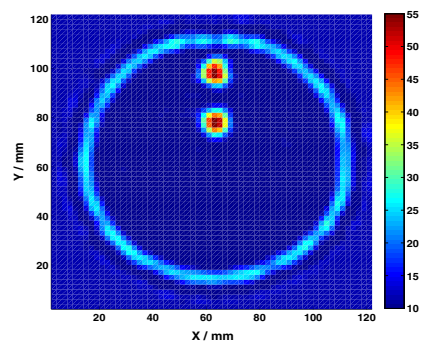

(a)

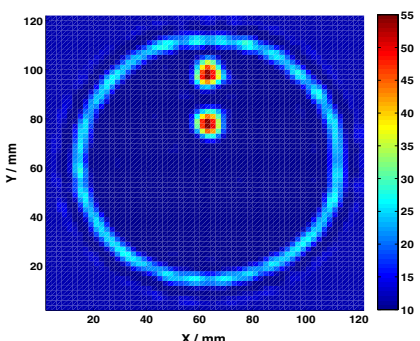

(b)

Figure 4: The noisy reconstructions when (a) the SNR is $15 \mathrm{~dB}$ and (b) the phase error is 10 degrees.

\section{Acknowledgments}

This work was supported in part by VINNOVA within the VINN Excellence Center Chase, and in part by SSF within the Strategic Research Center Charmant.

\section{References}

1. E. C. Fear and M. A. Stuchly, "Microwave detection of breast cancer," IEEE Trans. Microwave Theory Tech., vol. 48, no. 11, pp. 1854-1863, 2000.

2. E. C. Fear, S. C. Hagness, P. M. Meaney, M. Okoniewski and M. A. Stuchly, "Enhancing breast tumor detection with near-field imaging," Microwave Magazine, vol. 3, no. 1, pp. 48-56, 2002.

3. A. J. Surowiec, S. S. Stuchly, J. R. Barr, and A. Swarup, "Dielectric properties of breast carcinoma and the surrounding tissues," IEEE Trans. Biomed. Eng., vol. 35, no. 4, pp. 257-263, 1988.

4. S. Gabriely, R. W. Lau and C. Gabriel, "The dielectric properties of biological tissues: II. Measurements in the frequency range $10 \mathrm{~Hz}$ to $20 \mathrm{GHz}, "$ Phys. Med. Biol., vol. 41, no. 11, pp.2251-2269, 1996.

5. M. Lazebnik, D. Popovic, L. McCartney, C. Watkins, and etc., "A large scale study of the ultrawideband microwave dielectric properties of normal, benign and malignant breast tissues obtained from cancer surgeries," Phys. Med. Biol., vol. 52, no. 20, pp. 6093-6115, 2007.

6. P. M. Meaney, M. W. Fanning, and D. Li etc., "A clinical prototype for active microwave imaging of the breast," IEEE Trans. Microwave Theory Tech., vol. 48, no. 11, pp. 1841-1853, 2000.

7. A. Fhager, P. Hashemzadeh, and M. Persson, "Reconstruction quality and spectral content of an electromagnetic time-domain inversion algorithm," IEEE Trans. Biomed. Eng., vol. 53, pp. 1594-1604, 2006.

8. E. J. Bond, L. Xu, S. C. Hagness and B. D. Van Veen, "Microwave imaging via space-time beamforming for early detection of breast cancer," IEEE Trans. Antennas Propag., vol. 51, no. 8, pp. 1690-1705, 2003. 